QUANTUM DYNAMICS

BANACH CENTER PUBLICATIONS, VOLUME 120
INSTITUTE OF MATHEMATICS
POLISH ACADEMY OF SCIENCES
WARSZAWA 2020

\title{
A GRADED PULLBACK STRUCTURE OF LEAVITT PATH ALGEBRAS OF TRIMMABLE GRAPHS
}

\author{
PIOTR M. HAJAC \\ Instytut Matematyczny Polskiej Akademii Nauk \\ Sniadeckich 8, 00-656 Warszawa, Poland \\ ORCID:0000-0001-9764-5697_E-mail: pmh@impan.pl \\ ATABEY KAYGUN \\ Istanbul Technical University \\ Istanbul, Turkey \\ ORCID: 0000-0002-9672-6660_E-mail: kaygun@itu.edu.tr \\ MARIUSZ TOBOLSKI \\ Instytut Matematyczny Polskiej Akademii Nauk \\ Śniadeckich 8, 00-656 Warszawa, Poland \\ ORCID:0000-0001-7738-8515_E-mail: mtobolski@impan.pl
}

\begin{abstract}
Motivated by recent results in graph $\mathrm{C}^{*}$-algebras concerning an equivariant pushout structure of the Vaksman-Soibelman quantum odd spheres, we introduce a class of graphs called trimmable. Then we show that the Leavitt path algebra of a trimmable graph is gradedisomorphic to a pullback algebra of a subgraph Leavitt path algebra and the algebra of Laurent polynomials tensored with another subgraph Leavitt path algebra.
\end{abstract}

1. Introduction. The goal of this paper is to introduce and apply the concept of a trimmable graph. We begin by recalling the fundamental concepts of path algebras [6] and Leavitt path algebras [7, 3, 1, 2,. Then we define a trimmable graph, and prove our main result: There is a $\mathbb{Z}$-graded algebra isomorphism from the Leavitt path algebra of

2010 Mathematics Subject Classification: Primary 16S99; Secondary 46L55.

Key words and phrases: graph algebras, pullback diagrams, noncommutative dynamics. The paper is in final form and no version of it will be published elsewhere. 
a trimmable graph to an appropriate pullback algebra. The graph $\mathrm{C}^{*}$-algebraic version of this result is proven in [5], where it was used to analyze the generators of K-groups of quantum complex projective spaces.

\section{Leavitt path algebras}

Definition 2.1. A graph $Q$ is a quadruple $\left(Q_{0}, Q_{1}, s, t\right)$ consisting of the set of vertices $Q_{0}$, the set of edges $Q_{1}$, and the source and target maps $s, t: Q_{1} \rightarrow Q_{0}$ assigning to each edge its source and target vertex, respectively.

We say that a graph $Q^{\prime}=\left(Q_{0}^{\prime}, Q_{1}^{\prime}, s^{\prime}, t^{\prime}\right)$ is a sub-graph of a graph $Q=\left(Q_{0}, Q_{1}, s, t\right)$ iff $Q_{0}^{\prime} \subseteq Q_{0}, Q_{1}^{\prime} \subseteq Q_{1}$, and the source and target maps $s^{\prime}$ and $t^{\prime}$ are respective restrictionscorestrictions of the source and target maps $s$ and $t$. Furthermore, we say that two edges are composable if the end of one of them is the beginning of the other one. Now we can define a path in a graph as a sequence of composable edges. The length of a path is the number of edges it consists of, infinity included. We treat vertices as zero-length paths that begin and end in themselves.

Definition 2.2. Let $k$ be a field and $Q$ be a graph. The path algebra $k Q$ is the $k$-algebra whose underlying vector space has as its basis the set of all finite-length paths $\operatorname{Path}(Q)$. The product is given by the composition of paths when the end of one path matches the beginning of the other path. The product is defined to be zero otherwise.

One can check that the path algebra $k Q$ is unital if and only if the set of vertices $Q_{0}$ is finite. Then the unit is the sum of all vertices. It is also straightforward to verify that $k Q$ is $\mathbb{N}$-graded by the path length.

To define a Leavitt path algebra, we need ghost edges. For any graph $Q=\left(Q_{0}, Q_{1}, s, t\right)$, we create a new set $Q_{1}^{*}:=\left\{x^{*} \mid x \in Q_{1}\right\}$ and call its elements ghost edges. Now, the source and the target maps for the extended graph $\widehat{Q}:=\left(Q_{0}, Q_{1} \amalg Q_{1}^{*}, \hat{s}, \hat{t}\right)$ are defined by

$$
\hat{s}(x):=s(x), \quad \hat{s}\left(x^{*}\right):=t(x), \quad \hat{t}(x):=t(x), \quad \hat{t}\left(x^{*}\right):=s(x) .
$$

Definition 2.3. Let $k$ be a field and $Q$ a graph. The Leavitt path algebra $L_{k}(Q)$ of a graph $Q$ is the path algebra of the extended graph $\widehat{Q}$ divided by the ideal generated by the relations:

1. For all edges $x_{i}, x_{j} \in Q_{1}$, we have $x_{i}^{*} x_{j}=\delta_{i j} t\left(x_{i}\right)$.

2. For every vertex $v \in Q_{0}$ whose preimage $s^{-1}(v)$ is not empty and finite, we have

$$
\sum_{x \in s^{-1}(v)} x x^{*}=v .
$$

In other words, the Leavitt path algebra $L_{k}(Q)$ of a graph $Q$ is the universal $k$-algebra generated by the elements $v \in Q_{0}, x \in Q_{1}, x^{*} \in Q_{1}^{*}$, subject to the relations:

(L1) $v_{i} v_{j}=\delta_{i j} v_{i}$ for all $v_{i}, v_{j} \in Q_{0}$,

(L2) $s(x) x=x t(x)=x$ for all $x \in Q_{1}$,

(L3) $t(x) x^{*}=x^{*} s(x)=x^{*}$ for all $x^{*} \in Q_{1}^{*}$,

(L4) $x_{i}^{*} x_{j}=\delta_{i j} t\left(x_{i}\right)$ for all $x_{i}, x_{j} \in Q_{1}$, and

(L5) $\sum_{x \in s^{-1}(v)} x x^{*}=v$ for all $v \in Q_{0}$ such that $s^{-1}(v)$ is finite and nonempty. 
Furthermore, note that the $\mathbb{N}$-grading of the path algebra $k \widehat{Q}$ induces a $\mathbb{Z}$-grading of the Leavitt path algebra $L_{k}(Q)$ by counting the length of any ghost edge as -1 (see [3, Lemma 1.7]). Let us recall now the Graded Uniqueness Theorem [9, Theorem 4.8] that shows the importance of this grading. We will need it in the next section.

THEOREM $2.4([9])$. Let $Q$ be an arbitrary graph and $k$ be any field. If $A$ is a $\mathbb{Z}$-graded ring, and $f: L_{k}(E) \rightarrow A$ is a graded ring homomorphism with $f(v) \neq 0$ for every vertex $v \in Q_{0}$, then $f$ is injective.

3. Trimmable graphs. We are now ready for the main definition of the paper. Merely to focus attention, we assume henceforth that graphs are finite, i.e. that the set of vertices and the set of edges are both finite.

Definition 3.1. Let $Q$ be a finite graph consisting of a sub-graph $Q^{\prime}$ emitting at least one edge to an external vertex $v_{0}$ whose only outgoing edge $x_{0}$ is a loop (i.e. it ends where it begins). We call such a graph $v_{0}$-trimmable iff all edges from $Q^{\prime}$ to $v_{0}$ begin in a vertex emitting an edge that ends inside $Q^{\prime}$.

In symbols, a trimmable graph is described as follows:

$$
\begin{gathered}
Q_{0}=Q_{0}^{\prime} \cup\left\{v_{0}\right\}, \quad v_{0} \notin Q_{0}^{\prime}, \quad Q_{1}=Q_{1}^{\prime} \cup t^{-1}\left(v_{0}\right), \\
s^{-1}\left(v_{0}\right)=\left\{x_{0}\right\}, \quad t\left(x_{0}\right)=v_{0}, \quad t^{-1}\left(v_{0}\right) \backslash\left\{x_{0}\right\} \neq \emptyset, \\
\forall v \in s\left(t^{-1}\left(v_{0}\right) \backslash\left\{x_{0}\right\}\right): \quad s^{-1}(v) \backslash t^{-1}\left(v_{0}\right) \neq \emptyset .
\end{gathered}
$$

The conditions for a trimmable graph guarantee that, when we remove the distinguished vertex $v_{0}$, the resulting graph does not have a new sink. (A vertex is called a sink iff it emits no edge.) One can imagine a $v_{0}$-trimmable graph like this:

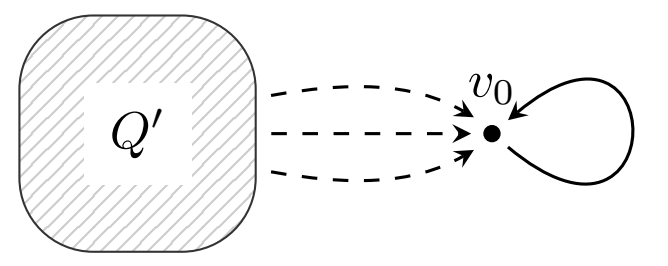

The following graph is a simple example of a $v_{0}$-trimmable graph:

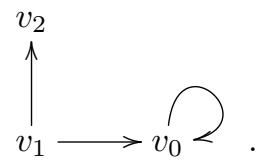

Note that the direction of the edge joining vertices $v_{1}$ and $v_{2}$ is important as the following graph is no longer $v_{0}$-trimmable:

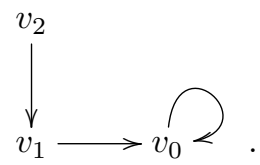


We need the trimmability conditions to guarantee the existence of maps given in the lemma below. This lemma is an algebraic incarnation of a graph- $\mathrm{C}^{*}$-algebraic lemma proved in [5]. The only difference between their proofs is that here, instead of using the Gauge Uniqueness Theorem [9, Theorem 4.8], we use the Graded Uniqueness Theorem [4. Theorem 2.3] (Theorem 2.4).

Lemma 3.2. Let $Q$ be a $v_{0}$-trimmable graph. Denote by $Q^{\prime \prime}$ the sub-graph of $Q$ obtained by removing the edge $x_{0}$. The following formulas define homomorphisms of algebras:

1. $\pi_{1}: L_{k}(Q) \rightarrow L_{k}\left(Q^{\prime}\right)$,

$$
\pi_{1}(\alpha):= \begin{cases}\alpha & \text { if } \alpha \in Q_{0}^{\prime} \cup Q_{1}^{\prime} \cup Q_{1}^{\prime *} \\ 0 & \text { otherwise. }\end{cases}
$$

2. $\pi_{2}: L_{k}\left(Q^{\prime \prime}\right) \rightarrow L_{k}\left(Q^{\prime}\right)$,

$$
\pi_{2}(\alpha):= \begin{cases}\alpha & \text { if } \alpha \in Q_{0}^{\prime} \cup Q_{1}^{\prime} \cup Q_{1}^{\prime *}, \\ 0 & \text { otherwise. }\end{cases}
$$

3. $f: L_{k}(Q) \rightarrow L_{k}\left(Q^{\prime \prime}\right) \otimes k\left[u, u^{-1}\right]$,

$$
f(\alpha):= \begin{cases}\alpha \otimes 1 & \text { if } \alpha \in Q_{0}, \\ v_{0} \otimes u & \text { if } \alpha=x_{0}, \\ v_{0} \otimes u^{-1} & \text { if } \alpha=x_{0}^{*}, \\ \alpha \otimes u & \text { if } \alpha \in Q_{1} \backslash\left\{x_{0}\right\}, \\ \alpha \otimes u^{-1} & \text { if } \alpha \in Q_{1}^{*} \backslash\left\{x_{0}^{*}\right\} .\end{cases}
$$

4. $\delta: L_{k}\left(Q^{\prime}\right) \rightarrow L_{k}\left(Q^{\prime}\right) \otimes k\left[u, u^{-1}\right]$,

$$
\delta(\alpha):= \begin{cases}\alpha \otimes 1 & \text { if } \alpha \in Q_{0}^{\prime}, \\ \alpha \otimes u & \text { if } \alpha \in Q_{1}^{\prime}, \\ \alpha \otimes u^{-1} & \text { if } \alpha \in\left(Q_{1}^{\prime}\right)^{*} .\end{cases}
$$

These morphisms are $\mathbb{Z}$-graded for the standard grading on $L_{k}(Q), L_{k}\left(Q^{\prime}\right), L_{k}\left(Q^{\prime \prime}\right)$, and the gradings on $L_{k}\left(Q^{\prime}\right) \otimes k\left[u, u^{-1}\right]$ and $L_{k}\left(Q^{\prime \prime}\right) \otimes k\left[u, u^{-1}\right]$ given by the standard grading of the rightmost tensorand. Furthermore, $\pi_{1}$ and $\pi_{2}$ are surjective, and $f$ and $\delta$ are injective.

4. A graded pullback structure. To prove the theorem of the paper, we need a general lemma along the lines of [8, Proposition 3.1]. We omit its routine proof.

Lemma 4.1. Let $A_{1}, A_{2}, B$ and $P$ be abelian groups. A commutative diagram of group homomorphisms

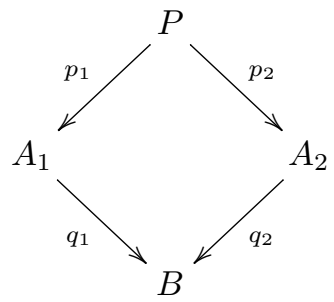


is a pullback diagram if and only if the following conditions hold:

$$
\begin{gathered}
\operatorname{ker}\left(p_{1}\right) \cap \operatorname{ker}\left(p_{2}\right)=\{0\} \\
q_{1}^{-1}\left(q_{2}\left(A_{2}\right)\right)=p_{1}(P), \\
p_{2}\left(\operatorname{ker}\left(p_{1}\right)\right)=\operatorname{ker}\left(q_{2}\right) .
\end{gathered}
$$

Recall that to prove that an algebra $P$ is a pullback algebra, one can proceed as follows. The first step is to establish the existence of a commutative diagram of algebra homomorphisms as above. This implies that $p_{1}$ and $p_{2}$ define an algebra homomorphism $p$ into the pullback algebra of $A_{1}$ and $A_{2}$ over $B$. Then one only needs to prove that the three conditions of Lemma 4.1 are satisfied to conclude that $p$ is an isomorphism. Note that (7) is equivalent to the injectivity of $p$, whereas the conjunction of (8) and (9) is equivalent to the surjectivity of $p$.

Much as Lemma 3.2, the theorem of the paper is an algebraic incarnation of a graph$\mathrm{C}^{*}$-algebraic theorem proved in [5]. This time, the only difference between their proofs is that here, instead of using [4, Lemma 3.1], we use [2, Lemma 2.4.1] (cf. [9, Lemma 5.6]).

THEOREM 4.2. Let $\pi_{1}, \pi_{2}, f$ and $\delta$ be as in Lemma 3.2. Then the commutative diagram

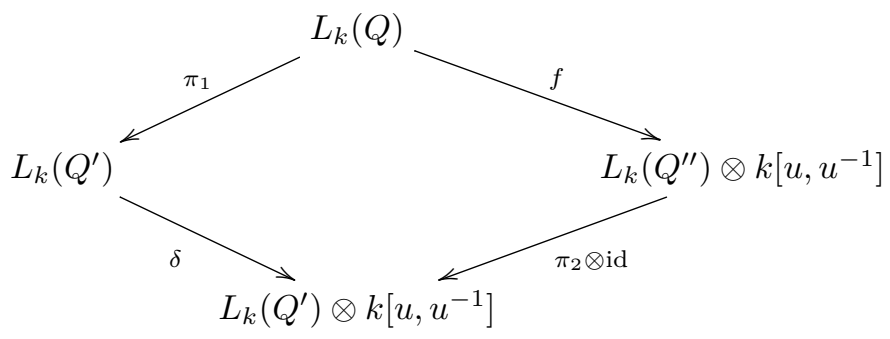

of graded algebra homomorphisms is a pullback diagram.

Representing pictorially the Leavitt path algebras by their respective graphs, the above diagram becomes:

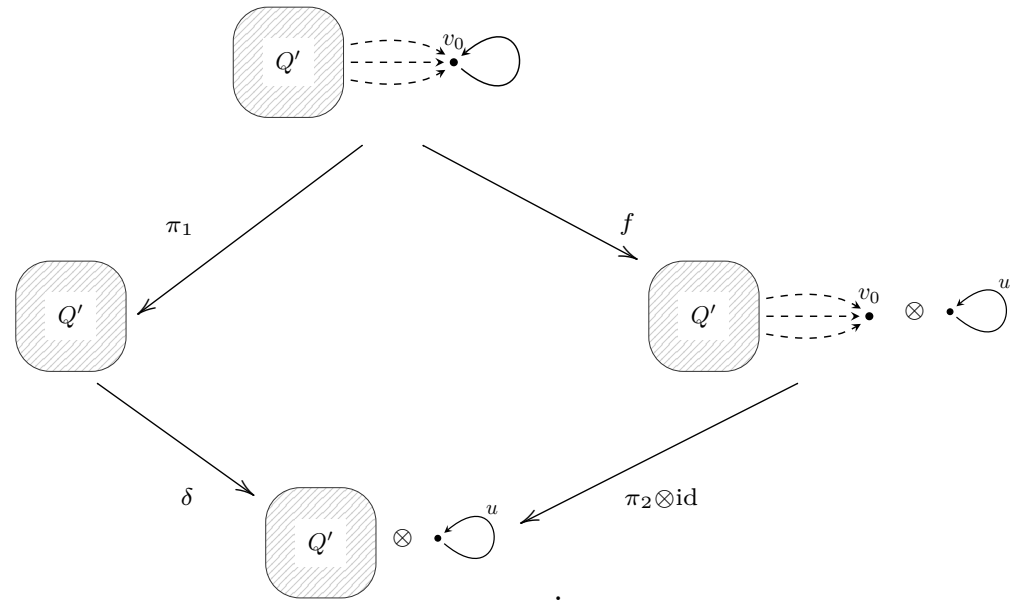


Note that the only non-standard map in this diagram is $f$. It can be described verbally by the assignment

$$
\begin{aligned}
\text { vertex } & \longmapsto \text { vertex } \otimes 1, \\
v_{0} \text {-emitted edge } & \longmapsto v_{0} \otimes u, \\
\text { other edge } & \longmapsto \text { other edge } \otimes u .
\end{aligned}
$$

Acknowledgments. The authors are very grateful to Francesco D'Andrea for sharing his mathematical insight with us, and for designing the pictures of graphs. It is also a pleasure to thank Søren Eilers, Adam Skalski, Wojciech Szymański and Anna Wysoczańska-Kula for discussions. This work was partially supported by NCN grant UMO-2015/19/B/ST1/03098.

\section{References}

[1] G. Abrams, Leavitt path algebras: the first decade, Bull. Math. Sci. 5 (2015), 55-120.

[2] G. Abrams, P. Ara, M. Siles Molina, Leavitt Path Algebras, Lecture Notes in Math. 2191, Springer, London, 2017.

[3] G. Abrams, G. Aranda Pino, The Leavitt path algebra of a graph, J. Algebra 293 (2005), 319-334.

[4] A. an Huef, I. Raeburn, The ideal structure of Cuntz-Krieger algebras, Ergodic Theory Dynam. Systems 17 (1997), 611-624.

[5] F. Arici, F. D'Andrea, P. M. Hajac, M. Tobolski, An equivariant pullback structure of trimmable graph $C^{*}$-algebras, J. Noncommut. Geom., to appear.

[6] I. Assem, D. Simson, A. Skowronski, Elements of the Representation Theory of Associative Algebras. Vol. 1. Techniques of Representation Theory, London Math. Soc. Stud. Texts 65, Cambridge Univ. Press, Cambridge, 2006.

[7] W. G. Leavitt, The module type of a ring, Trans. Amer. Math. Soc. 103 (1962), 113-130.

[8] G. K. Pedersen, Pullback and pushout constructions in $C^{*}$-algebra theory, J. Funct. Anal. 167 (1999), 243-344.

[9] M. Tomforde, Uniqueness theorems and ideal structure for Leavitt path algebras, J. Algebra 318 (2007), 270-299. 